\title{
Performance evaluation of high mobility OFDM channel estimation techniques
}

\author{
Maryam K. Abboud, Bayan M. Sabbar \\ Department of Information and Communication Engineering, AL-Nahrain University, Iraq
}

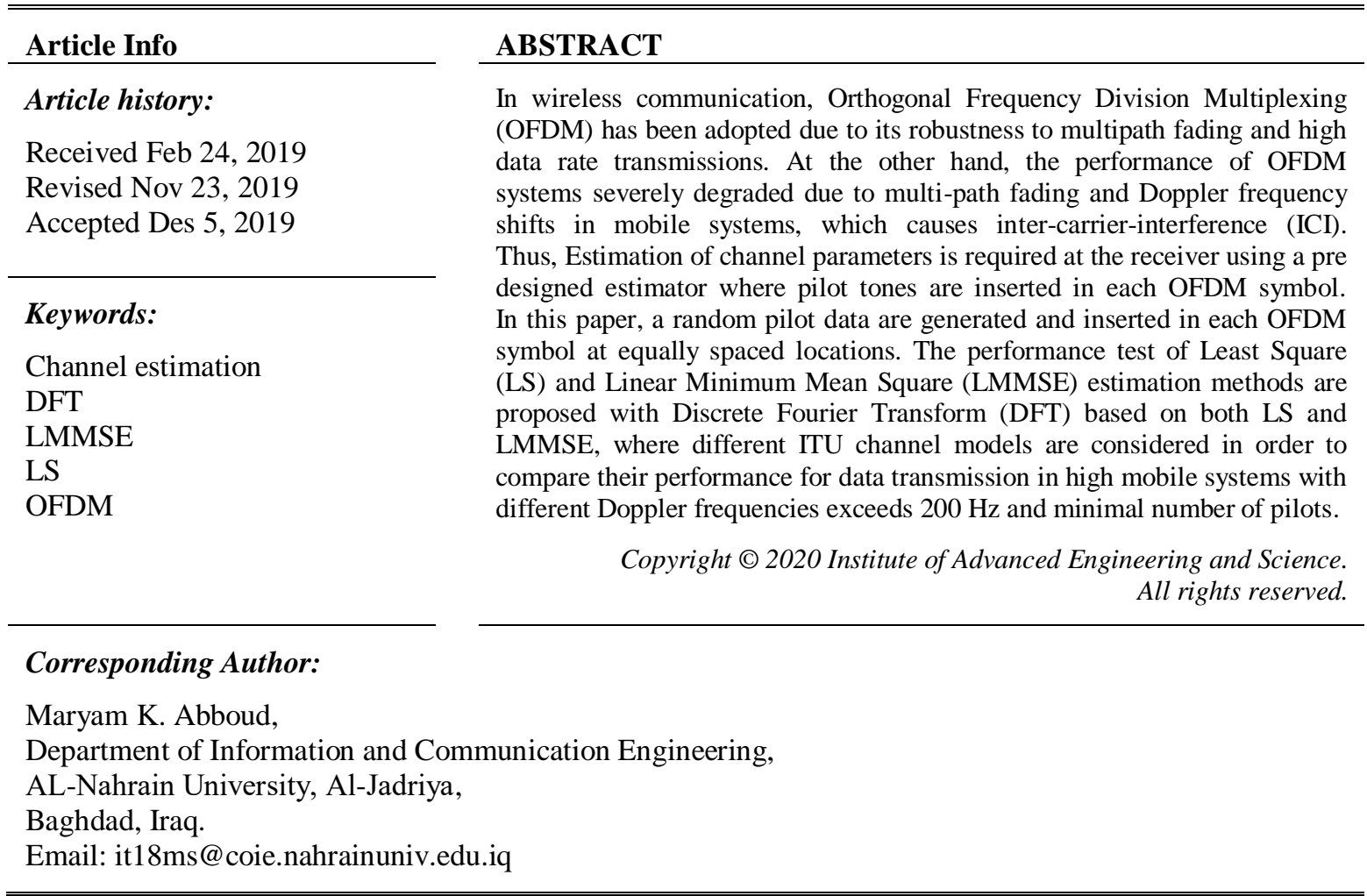

\section{INTRODUCTION}

Recent mobile applications require high and reliable data transmissions, thus, OFDM has been deployed in several applications due to its spectral efficiency and robustness to multipath fading. High speed data transmissions severs from frequency selective fading which causes a serious Inter Symbol Interference (ISI) between the transmitted symbols. OFDM can overcome this problem with high data rates. Channel estimation is an important part of OFDM system and it is an essential process to recover the channel impulse response $[1,2]$. In high mobility systems, the resulting high Doppler shifts leads to performance degradation in channel estimation accuracy [3].

Majority of communication systems adopt the coherent detection in order to pursue better transmission performance through channel estimation of Channel State Information (CSI). Several techniques are adopted for the purpose of channel estimation in OFDM systems. LS and LMMSE estimation methods are the most popular pilot based channel estimation techniques [4, 5]. A performance comparison of LS and LMMSE is proposed in [4], where the two methods are simulated and LMMSE performs better than LS with Binary Phase Shift Keying (BPSK) modulation. A pilot insertion method was proposed in [6] to test the performance of LS estimation in OFDM system, where it based on a combination of block and comb types using linear and cubic spline interpolation [7].

Discrete Fourier Transform (DFT) based pilot insertion method is one of estimation techniques introduced in several publications in order to improve estimation performance. Since DFT estimation based on noise cancellation in time domain using Fast Fourier Transform (FFT), it represents a low complex method with performance depends on tested channel environments [8-10]. An improved DFT method was 
proposed in [11] in order to enhance estimation performance close to LMMSE with lower complexity, where all samples outside the cyclic prefix are considered noisy and set to zero. In [12], a conventional and improved DFT methods were proposed and only significant components within cyclic prefix length are selected while the noise components are ignored. The research based on selecting different number of iterations in each trial, where the one gives best performance is evaluated. The proposed work test the environments effects on channel estimation techniques with different Doppler frequencies not exceeding $33 \mathrm{~Hz}$. In [13], OFDM estimation algorithms were tested for a time variant channel with different pilot insertion schemes. Block type, comb type, and a hybrid pilot insertion scheme was proposed. The test of all schemes shows that the comb type pilot insertion method performs better than block type and the hybrid scheme yields a good tradeoff between them.

With the revolution in communication systems, several estimation techniques are proposed in order to enhance system performance at mobility situations. The enhancement cost increased as number of pilots increased where bandwidth efficiency degraded [14]. A lower bound of pilot's number was targeted by [14-15], which stated that, in order to achieve acceptable BER performance and good throughput and if the channel length is known or estimated, the number of pilots Np must be greater or equal to the channel length assuming equally-spaced and equal power pilot tones. Thus, the target of this paper is to evaluate the performance of conventional estimation techniques with minimum number of pilots as required by [14], with high Doppler frequencies, in order to save bandwidth using a predetermined pilot locations.

The rest of the paper is organized as follows. A brief background on channel estimation and the criteria of estimation techniques was proposed in section 2. Clarifying such model together with other system parameters and specifications proposed in section 3. Performance evaluation in the form of Bit Error Rate (BER) is then presented in sections 4. Finally the main concluding remarks are given in section 5.

\section{BACKGROUND}

Channel estimation is an effective tool to predict the behavior of the channel and it is adopted at the receiver side. Training based channel estimation and blind channel estimation are the two main methods may be used for this purpose, where, training based channel estimation is the popular and widely used according to its ability to obtain an effective CSI by fully utilizing its pilots and training sequences [13, 16-18]. A brief description for Least Square (LS), Linear Minimum Mean Square (L MMSE), and Discrete Fourier Transform (DFT) techniques are reviewed in the next subsections.

\subsection{Least square (LS)}

It is the simplest channel estimation method, where the transmitted signals at pilot subcarriers are used to estimate the channel impulse response. The estimated channel taps are then used to recover the transmitted data $[4,19]$.

$$
H_{L S}[k]=\frac{Y_{p}[k]}{X_{p}[k]} \quad 1<k<N_{p}
$$

$H_{L S}[k]$, the estimated channel response at $k^{\text {th }}$ pilot subcarrier using LS.

$X_{p}[k]$, the transmitted symbol at $k^{\text {th }}$ pilot subcarrier.

$Y_{p}[k]$, the received symbol at $k^{\text {th }}$ pilot subcarrier.

$N_{p}$, the number of pilots.

\subsection{Linear minimum mean square (LMMSE)}

It is a more complex channel estimation method as compared to LS method. LMMSE depends on minimizing the error between the transmitted and received signals through the expression below [12, 20];

$$
H_{M M S E}=H_{L S} \times W
$$

where $\mathrm{W}$ is a weighted matrix and it is given by;

$$
W=R_{H H L S} R_{H L S H L}^{1}
$$

and hence, the error value computed by [12];

$$
e=H-H_{M M S E}
$$


where:

$H_{M M S E}$, the estimated channel response using MMSE

$R_{H L S H L}^{1}$, the auto-correlation matrix of HLS

$R_{H H L S}$, the cross-correlation matrix of the true channel and estimated one

$H$, represents the true channel coefficients.

\subsection{Discrete fourier transform (DFT)}

The Discrete Fourier Transform (DFT) based channel estimation method is introduced to improve the performance of LS and MMSE methods. This method is used for noise reduction where it eliminate the noise outside the maximum channel delay [21].

$$
\operatorname{IDFT}\left(H_{L S}\right)=h \operatorname{DFT}[n]=h[n]+z[n] \quad n=0,1 \ldots, N-1
$$

where:

$H_{L S}[\mathrm{n}]$, the estimated channel response at nth subcarrier using LS

$\mathrm{h}[\mathrm{n}]$, the sampled impulse response of the channel

$\mathrm{z}[\mathrm{n}]$, the noise component in the estimated channel

\section{SYSTEM MODLE}

Orthogonal frequency division multiplexing is a promising multi carrier modulation scheme. It is a widely used technology in rich multi path wireless communications due to its ability to overcome the ISI problem [22]. It also provide a simple equalization process in frequency domain [2, 23, 24]. The proposed OFDM based channel estimation model is defined as shown in Figure 1(a). Where different number of pilots are inserted within the transmitted OFDM symbol for the purpose of retrieving the CSI with a simple frequency equalization process.

As mentioned before in section I, the number of pilots is constrained by the relationship [14, 15, 25];

$$
N_{p} \geq L^{\wedge}
$$

where $L^{\wedge}$ is the expected channel length

Since CP was chosen to cover the maximum channel delay, in the proposed simulations, the number of pilots are selected to be greater than or equal to the $\mathrm{CP}$ length in the presence of Doppler shifts.

$$
N_{p} \geq L_{c p}
$$

where they are assumed to have an equal power and inserted at equally spaced locations in each OFDM symbol as shown in Figure 1(b).

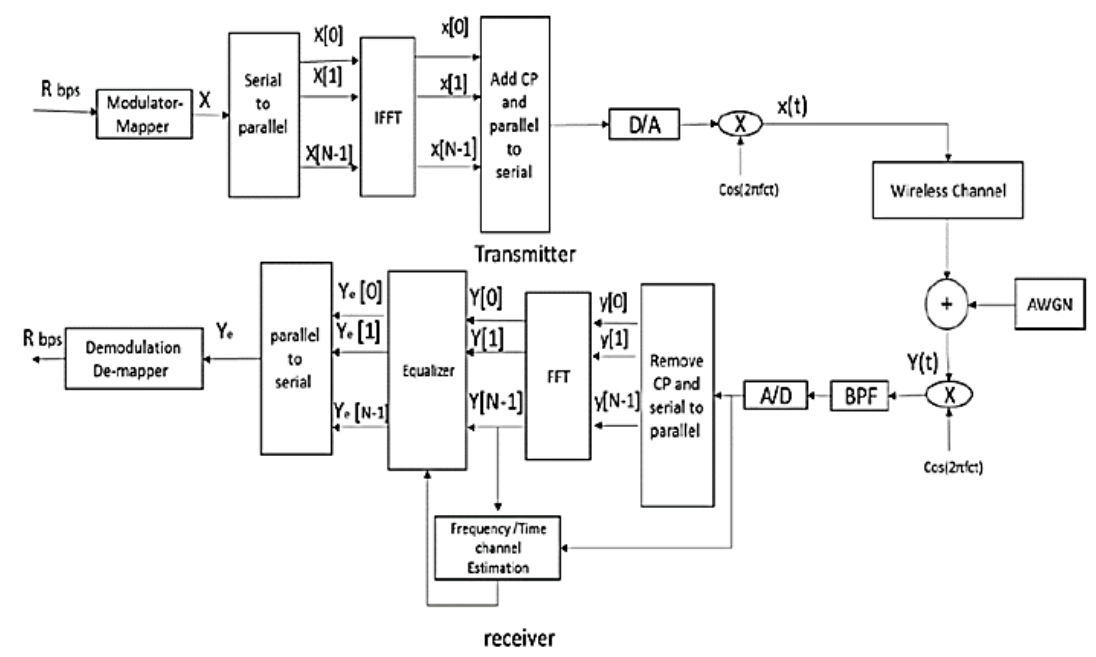

(a) 


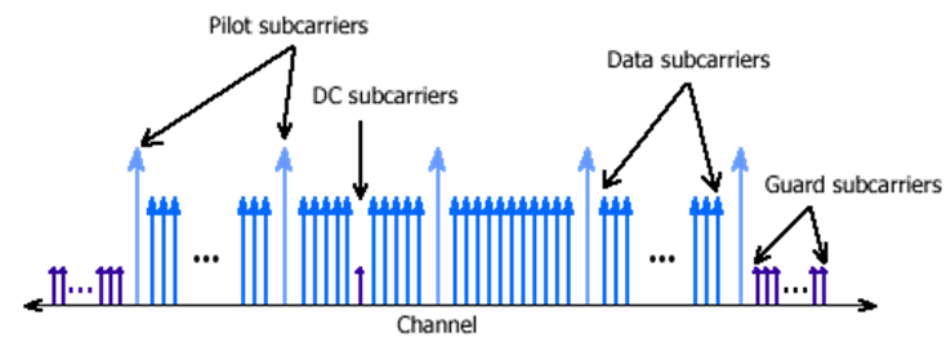

(b)

Figure 1. (a) OFDM Communication System [6], (b) Pilot and data subcarriers locations

A set of commonly used channels in mobile communication systems was specified in ITU-R recommendation M.1225. Three channel environments are proposed in this specification; Indoor, Pedestrian, and Outdoor. For each of these specified environments, a multipath tap delay profile is specified as shown in Table 1 [26].

Table 1. ITU channel models [26]

\begin{tabular}{ccccccc}
\hline & \multicolumn{2}{c}{$\begin{array}{c}\text { Indoor Environment } \\
(\mathrm{A})\end{array}$} & \multicolumn{2}{c}{$\begin{array}{c}\text { Pedestrian Environment } \\
(\mathrm{B})\end{array}$} & \multicolumn{2}{c}{$\begin{array}{c}\text { Outdoor Environment } \\
(\mathrm{A})\end{array}$} \\
\hline Tap No. & Delay $(\mathrm{ns})$ & Power $(\mathrm{dB})$ & Delay $(\mathrm{ns})$ & Power $(\mathrm{dB})$ & Delay $(\mathrm{ns})$ & Power dB) \\
1 & 0 & 0 & 0 & 0 & 0 & 0 \\
2 & 50 & -3 & 200 & -0.9 & 310 & -1.5 \\
3 & 110 & -10 & 800 & -4.9 & 710 & -9.0 \\
4 & 170 & -18 & 1200 & -8.0 & 1090 & -10.0 \\
5 & 290 & -26 & 2300 & -7.8 & 1730 & -15.0 \\
6 & 310 & -32 & 3700 & -23.9 & 2510 & -20.0 \\
\hline
\end{tabular}

\section{SIMULATION TESTS AND RESULTS}

The OFDM system of Figure 1(a) was tested with different estimation techniques which were described briefly in the previous section. In addition to AWGN, the test considers a Rayleigh fading channel with delay and power vectors standardized by ITU channel models of Table 1. For each pilot aided estimation method tested, the pilot insertion method consider an equally spaced locations with spline interpolation. The results are plotted in the form of Bit Error Rate (BER) versus Signal to Noise Ratio (SNR), where SNR is determined by the corresponding $\left(\mathrm{E}_{\mathrm{b}} / \mathrm{N}_{\mathrm{o}}\right)$ in $\mathrm{dB}$. The required system parameters of OFDM system, Doppler shift $\left(F_{d}\right)$, and data rate $\left(R_{s}\right)$ are shown in Table 2 . Where, when $F_{d}=0, N_{p}=8<L_{c p}$, while when $F_{d}>0, N_{p}=16$ which is the considered length of $L_{c p}$.

Table 2. System parameters

\begin{tabular}{ll}
\hline Parameter & Value \\
\hline Number of transmitted bits & 64000 \\
Modulation & BPSK \\
Rate b/sec. & Variable \\
OFDM Subcarriers & 64 \\
Number of pilots $\left(N_{p}\right)$ & 8,16 \\
Cyclic prefix length $\left(L_{c p}\right)$ & 16 \\
Carrier Frequency $(\mathrm{fc})$ & $2.4 \mathrm{GHz}$ \\
Maximum Doppler shift $\left(F_{d}\right) \mathrm{Hz}$ & $0,5,250$ \\
\hline
\end{tabular}

In Figure 2, Practical examination approved theoretical results of OFDM simulation over AWGN channel. LS, LMMSE, DFT based LS, and DFT based LMMSE estimation algorithms are tested over ITU-Indoor channel model as shown in Figure 3 with zero Doppler shift and $10 \mathrm{Kbps}$ data rate, where 8 pilots are inserted at equally spaced locations along the OFDM symbol. For a BER of $10^{-3}$, the LMMSE test shows an improvement in SNR by about $0.5 \mathrm{~dB}$ over LS method, while the DFT-LS gives a $2 \mathrm{~dB}$ improvement in SNR over the LS tests. At the same channel environments, $1.8 \mathrm{~dB}$ improvement in SNR achieved for DFT-LMMSE over traditional LMMSE tests. For the case where DFT-LS compared to DFTLMMSE, the tests shows that DFT-LMMSE performs better than DFT-LS by about $0.25 \mathrm{~dB}$. 


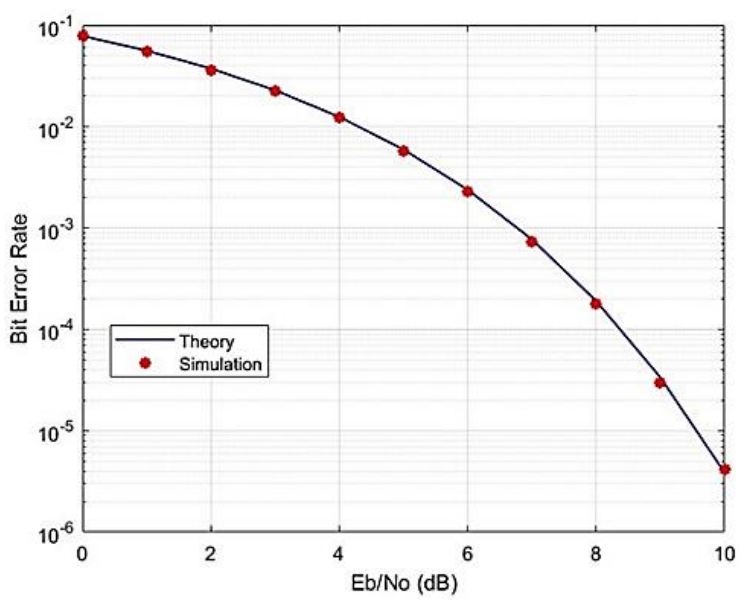

Figure 2. Performance evaluation of OFDM system in AWGN channel

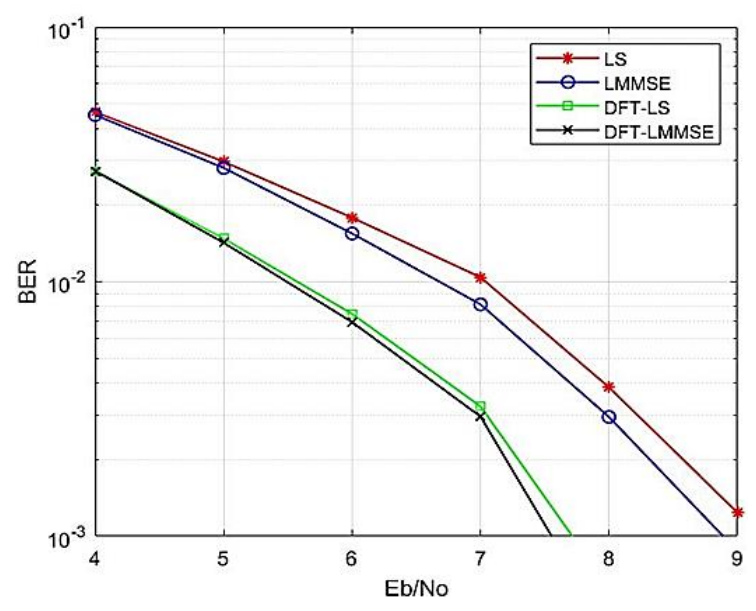

Figure 3. Performance evaluation of OFDM estimation in ITU-indoor channel

In Figure 4, the performance of the same methods are tested over ITU- Pedestrian channel with 16 pilots, data rate of $2.5 \mathrm{Kbps}$, and a Doppler shift of $5 \mathrm{~Hz}$. At BER of $10^{-2}$, the LMMSE gives an improvement in SNR by about $5.1 \mathrm{~dB}$ over LS method. At the other hand, a DFT-LS improve the performance of the traditional LS method by about $7.4 \mathrm{~dB}$. However, DFT-LMMSE improves the traditional LMMSE performance by about $3.3 \mathrm{~dB}$. As a consequences, DFT-LMMSE outperforms DFT-LS performance by about $1 \mathrm{~dB}$ at the same error rate value.
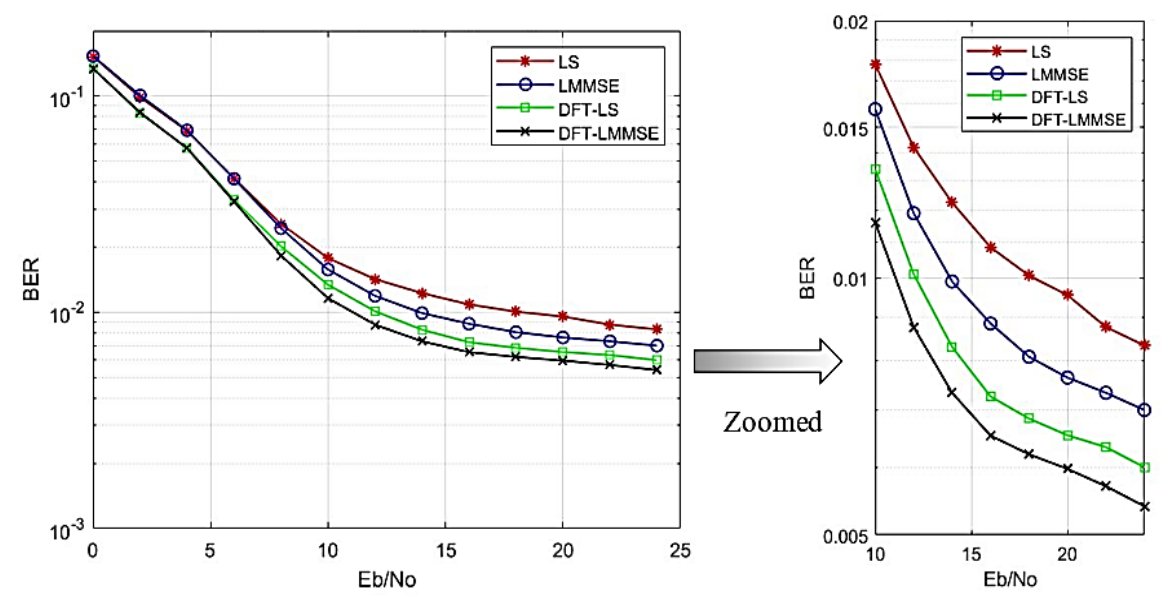

Figure 4. Performance evaluation of OFDM estimation in ITU- pedestrian channel

The same methods are tested over ITU-Outdoor channel to characterize their performance with high Doppler shift. In Figure 5, all presented estimation methods are tested with Doppler shift of $250 \mathrm{~Hz}$, 16 pilots, and $250 \mathrm{Mbps}$ data rate. For a BER of $10^{-2}$, LMMSE outperforms LS estimation method by about $9 \mathrm{~dB}$ and DFT-LS improves the performance of the traditional LS by about $11.2 \mathrm{~dB}$. Also, the performance of LMMSE is improved with DFT-LMMSE by about $6 \mathrm{~dB}$, where a $3.8 \mathrm{~dB}$ improvement achieved with DFT-LMMSE over DFT-LS method.

It is clear that in the presence of high Doppler shift, the estimation performance will degraded. This performance can be enhanced through increasing the number of pilots in each OFDM symbol which will decrease the bandwidth efficiency of the system. At the other hand, the proposed tests shows that even the Doppler frequency increased, an almost acceptable performance can be achieved with lower number of pilots equals to the channel length, and hence improve bandwidth utilization for bandwidth efficient applications. 
As a final assessment of results, a little compromising between SNR gain and implementation complexity, LS channel estimation method characterized by a low complexity implementation as compared to LMMSE and DFT-LMMSE. While the performance of DFT-LMMSE better than LS with SNR gain by about; $2.25 \mathrm{~dB}$ for $10^{-3}$ error rate with zero Doppler shift in ITU-Indoor channel, $8.4 \mathrm{~dB}$ for $10^{-2}$ error rate with $5 \mathrm{~Hz}$ Doppler shift in ITU- Pedestrian channel, and $15 \mathrm{~dB}$ for $10^{-2}$ error rate with $250 \mathrm{~Hz}$ Doppler shift in ITU- Outdoor channel.
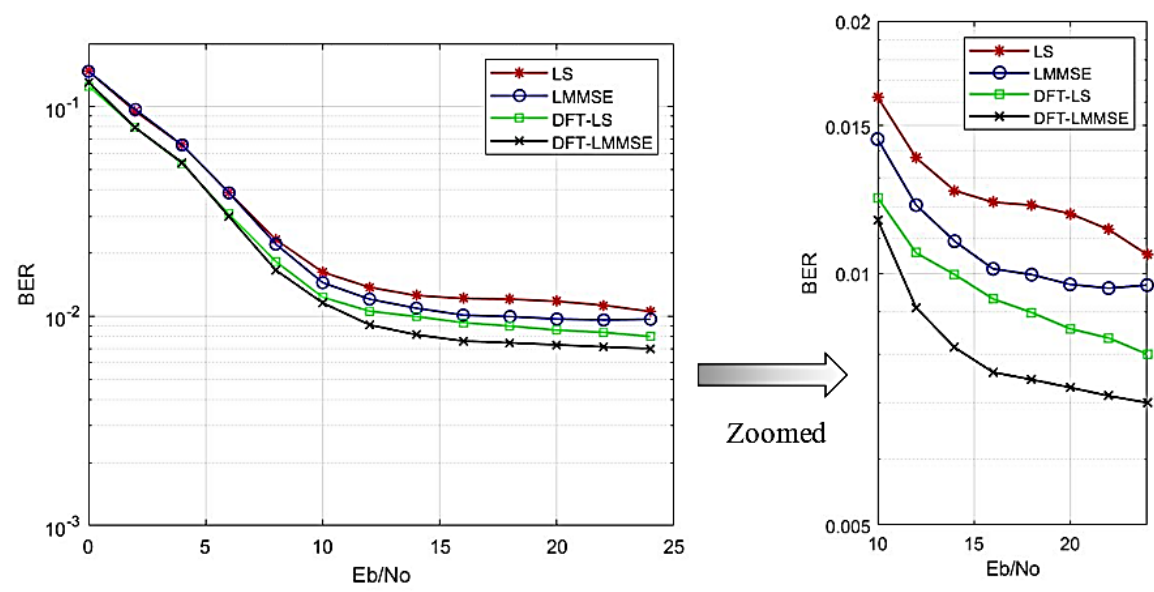

Figure 5. Performance evaluation of OFDM estimation in ITU-outdoor channel

\section{CONCLUSION}

Channel estimation process is an effective part of OFDM system especially in fading environment where channel statistics changes over time, and in the presence of Doppler shift the data transmission is hardly affected. In the proposed work, ITU channel models are considered to examine the suitability of different channel estimation techniques for high mobility environment in the presence of deep fading and high Doppler shifts, where minimal number of random pilots are inserted in each OFDM symbol at equally spaced locations. In such environment, the tests shows an improvement in the performance of estimation techniques when DFT adopted, where this improvement exceeds $11 \mathrm{~dB}$ and $15 \mathrm{~dB}$ for DFT-LS and DFTLMMSE over conventional LS method respectively with $250 \mathrm{~Hz}$ Doppler shift where the mobility reaches $112 \mathrm{~km} / \mathrm{h}$, a BER of $10^{-2}$, and 16 pilots. As a future work, a trading off between estimation complexity and BER improvement will be considered with high Doppler frequencies and reduced number of pilots.

\section{REFERENCES}

[1] Y. Li, L. J. Cimini, N. R. Sollenberger, "Robust Channel Estimation for OFDM Systems with Rapid Dispersive Fading Channels," IEEE Transactions on Communications, vol. 46, no. 7, 1998.

[2] T. Hwang and C. Yang, "OFDM and Its Wireless Applications: A Survey," IEEE Transaction on Vehicular Technology, vol. 58, no. 4, pp. 1673-1694, 2009.

[3] A. Dowler, A. Doufexi, and A. Nix, "Performance Evaluation of Channel Estimation Techniques for a Mobile Fourth Generation Wide Area OFDM System," IEEE 56th Vehicular Technology Conference, VTC Fall- 2002.

[4] S. M. Patil and A.N Jadhav, "Channel Estimation Using LS and MMSE Estimators," International Journal on Recent and Innovation Trends in Computing and Communication, vol. 2, no. 3, pp. 51-55, 2014.

[5] B. Deng and W. Wang, "Low complexity channel estimation method of MIMO-OFDM for LTE downlink," IEEE 7th International Conference on Wireless Communication, Networking and Mobile Computing (WiCOM), pp. 1-4, 2011.

[6] V. Kanwar, D. Sharma, H. Thakur, "Performance Evaluation of Block Type and Comb Type Channel Estimation for OFDM System under Various Modulation Techniques," IOSR Journal of Engineering (IOSRJEN), vol. 3, pp. 4, Apr. 2013.

[7] H. M. Mahmoud, A. S. Mousa, R. Saleem, "Channel Estimation Based in Comb-Type Pilots Arrangement for OFDM System over Time Varying Channel," Journal of Networks, vol. 5, no. 7, 2010.

[8] C. Kaur and R. Kaur, "Discrete Fourier Transform based Channel Estimation Scheme for MIMO-OFDM Communication System," International Journal of Engineering Research and Applications (IJERA), vol. 2, no. 3, pp. 2612-2616, 2012.

[9] E. Singh, "A DFT based channel estimation technique in orthogonal-frequency division-multiplexing (OFDM): A Review," International Journal of Recent Research Aspects ISSN, vol. 3, pp. 1, 2016. 
[10] Haifang and S. Yin, "An Efficient Iterative DFT-BASED Channel Estimation for MIMO-OFDM System on Multipath Channels," IEEE Third International Conference on Communication and Networking in China, 2008.

[11] J. Wang and Y. Qiu, "A new DFT based Channel Estimation Method for OFDM Systems over Multipath Channels," National Conference on Information Technology and Computer Science (CITCS 2012), 2012.

[12] N. A. Mansour, A. A. Kadhim, "Improved DFT - Based Channel Estimation Techniques for OFDM Signal," International Journal of Engineering Research, vol. 5, no. 12, pp. 922-926, 2016.

[13] Z. Tang and G. Leus, "Pilot Schemes for Time-Varying Channel Estimation OFDM Systems," IEEE, 2007.

[14] M. Imani, H. Bakhshi, "The Tight Bound for the Number of Pilots in Channel Estimation for OFDM Systems," Communications and Network, 2012.

[15] O. Ferreira1, E. Vale, J. R. Bello, "Optimal Number of Pilots for OFDM Systems," IOSR Journal of Electronics and Communication Engineering, vol. 8, no. 6, Dec. 2013.

[16] V. K. Gupta, S. Vijay, "A Summative Comparison of Blind Channel Estimation Techniques for Orthogonal Frequency Division Multiplexing Systems," International Journal of Electrical and Computer Engineering (IJECE), vol. 8, no. 5, pp. 2744-2752, 2018.

[17] S. Atoui, N. Doghmane, S. Afifi, "Blind frequency offset estimator for OFDM systems," TELKOMNIKA (Telecommunication, Computing, Electronics and Control), vol. 17, no. 6, pp. 2722-2728, 2019.

[18] Sakina A., Noureddine D., Saddek A., "Blind frequency offset estimator for OFDM systems," TELKOMNIKA (Telecommunication Computing Electronics and Control), vol. 17, no. 6, pp. 2722-2728, 2019.

[19] S. Pyla, K. P. Raju, N. B. Subrahmanyam, "Performance analysis of adaptive filter channel estimated MIMO OFDM communication system," International Journal of Electrical and Computer Engineering (IJECE), vol. 8, no. 5, pp. 3829-3838, 2018.

[20] A. Zaier, R. Bouallègue, "Channel Estimation Study for Block-Pilot Insertion in OFDM Systems Under Slowly Time Varying Conditions," International Journal of Computer Networks \& Communications (IJCNC), vol. 3, no. 6, 2011.

[21] H. Zhu, Y. Ge and X. Chen, "DFT-based Adaptive Channel Estimation for OFDM Systems," IEEE 16th International Conference on Communication Technology (ICCT), pp. 515-517, Oct. 2015.

[22] Bin Jiao, Jitian Xiao, Jinlin Wang, "Analysis of channel estimation error of OFDM systems in rayleigh fading," IEEE, 2005.

[23] D. Madan, Monika, V. Arora, "Equalization \& Channel Estimation of Block \& Comb Type Codes," International Journal of Innovative Research in Advanced Engineering (IJIRAE), vol. 1, no. 6, 2014

[24] S. Singh, S. S. Gill, "Performance Evaluation of Channel Estimation in OFDM System for Different QAM and PSK Modulations," International Journal of Electrical and Computer Engineering (IJECE), vol. 1 no. 2, pp. 140-150, 2011.

[25] Z. Tang, G. Leus, "Pilot Schems for Time-Varying Channel Estimation in OFDM Systems," IEEE, 2007.

[26] S. Ahmadi, R. Srinivasan, H. Choi, J. Park, J. Cho and D. Park, "Channel Models for IEEE 802.16m Evaluation Methodology Document," IEEE C802.16, Mar. 2007. 